\title{
Generalised Vector Precoding Design Based on the MBER Criterion for Multiuser Transmission
}

\author{
W. Yao, S. Chen and L. Hanzo \\ School of Electronics and Computer Science, University of Southampton, SO17 1BJ, UK \\ $\{$ wy07r, sqc, lh\}@ecs.soton.ac.uk, http://www-mobile.ecs.soton.ac.uk
}

\begin{abstract}
A generalised vector precoding (VP) design based on the minimum bit error rate (MBER) criterion is proposed for multiuser transmission in the downlink of a multiuser system where the base station (BS) equipped with multiple transmit antennas communicates with single-receive-antenna mobile station (MS) receivers each having a modulo detection device. Our transmit preprocessing scheme generates the effective symbol vector based on the MBER criterion, given the knowledge of the channel state information and the current information symbol vector to be transmitted. The proposed MBER based generalised VP algorithm is shown to outperform even the powerful minimum mean-square-error VP benchmark, particularly for rankdeficient systems where the number of BS's transmit antennas is smaller than the number MSs supported.
\end{abstract}

\section{INTRODUCTION}

The family of well-studied multiuser detectors (MUD) was designed for mitigating the multiuser interference at the base station (BS), which is imposed by the uplink (UL) transmissions of the mobile stations (MSs) to the BS. It is however less feasible to employ MUDs in the downlink (DL) of a space-division multiple-access (SDMA) system at the MSs. In order to facilitate the employment of a low-complexity, highpower-efficiency single-user receiver, the transmitted multiuser DL signals may be pre-processed at the BS, leading to the concept of multiuser transmission (MUT) [1]. More explicitly, the MUT requires the knowledge of each user's unique channel impulse response (CIR) for differentiating the different users' transmissions. For time division duplex systems, the UL CIRs measured at the BS may be exploited for subsequent DL preprocessing owing to the channel's reciprocity. However, the CIR has to be explicitly fed back from the MS's receivers to the BS transmitter in frequency division duplex systems, where the UL and DL CIRs are different. MUT schemes can be divided into the two groups, namely, linear and nonlinear MUT schemes.

The well-known linear MUT methods include the zeroforcing (ZF) and the minimum mean-square-error (MMSE) schemes [2], [3], which have an appealing simplicity, but they exhibit a limited bit error rate (BER) performance. Various linear MUT schemes based on the minimum BER (MBER) criterion [4]-[8] were proposed in order to improve the achievable BER performance. Recently, a low-complexity particle swarm optimisation (PSO) aided linear MBER MUT design was proposed in [9]. Nonlinear MUT techniques are capable of approaching the rate region of dirty paper coding [10]. The family of vector precoding (VP) schemes [11]-[14], where each receiver employs a modulo device, is capable of significantly outperforming linear MUT techniques. To elaborate a little further, the VP of [11] is based on the ZF criterion, while the powerful MMSE VP solution was derived in [12]. By contrast, the MMSE VP of [15] dispenses with the employment of the modulo device of [11]. An improved MMSE VP schemes based on the MBER criterion was developed in [16].

Against this background, in this paper, we consider an advanced VP design based on the MBER criterion. Our contributions are: (A) To the best of our knowledge, no VP algorithm was proposed in the literature based directly on the minimisation of the BER, hence we propose the MBER-based generalised VP algorithm. (B) Instead of the two-step process of generating the linear precoding matrix and perturbation vector of [12], we generate the effective symbol vector to be transmitted by directly minimising the BER criterion by exploiting the knowledge of the information symbol vector and the channel matrix. (C) Since the resultant design constitutes a non-convex continuous-valued optimisation problem, we adopt the computationally efficient PSO algorithm [17][19] for creating the generalised MBER VP design. (D) We demonstrate that the proposed MBER-based generalised VP algorithm outperforms even the powerful nonlinear MMSE VP benchmark proposed in [12], especially for rank-deficient systems, where the number of BSs transmit antennas is lower than the number MSs supported. (E) Our complexity study demonstrates that the complexity imposed by the generalised MBER VP design is no higher than twice that of the MMSE VP solution.

\section{SYSTEM MODEL}

The DL of a SDMA system is depicted Fig. 1, where the BS is equipped with $N$ DL transmit antennas and transmits over frequency-flat fading channels to $K$ non-cooperative MSs,

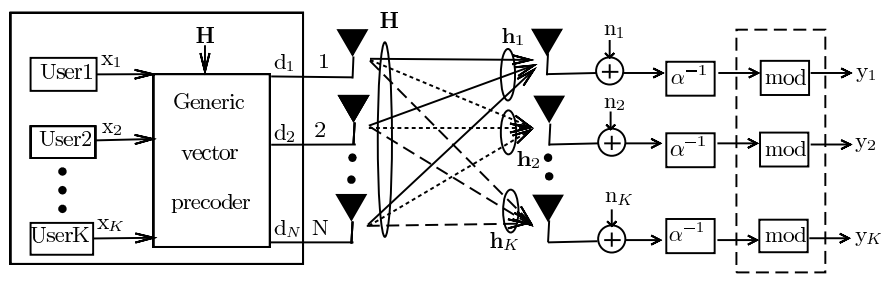

Fig. 1. Schematic diagram of the SDMA system's DL using preprocessing at the BS. The MUT-aided system employs $N$ transmit antennas at the BS to communicate with $K$ non-cooperative MSs each with a modulo device. 
each employing a single receive antenna and a modulo device. The DL channel matrix $\mathbf{H}$ of the system is given by

$$
\mathbf{H}=\left[\begin{array}{llll}
\mathbf{h}_{1} & \mathbf{h}_{2} & \cdots & \mathbf{h}_{K}
\end{array}\right],
$$

where $\mathbf{h}_{k}=\left[\begin{array}{ll}h_{1, k} & h_{2, k} \cdots h_{N, k}\end{array}\right]^{T}, 1 \leq k \leq K$, is the $k$ th user's spatial signature. The independent CIR taps $h_{i, k}$, for $1 \leq k \leq K$ and $1 \leq i \leq N$ obey the complex-valued Gaussian distribution having $E\left[\left|h_{i, k}\right|^{2}\right]=1$, where $E[\bullet]$ denotes the expectation. The information symbol vector to be transmitted to the $K \mathrm{MSs}$ is given by $\mathbf{x}=\left[\begin{array}{ll}x_{1} & x_{2} \cdots x_{K}\end{array}\right]^{T}$, where $x_{k}$ denotes the information symbol destined for the $k$ th MS. Given $\mathbf{x}$ and $\mathbf{H}$, the generic VP generates the continuous-valued effective symbol vector of $\mathbf{d}=\left[\begin{array}{ll}d_{1} & d_{2} \cdots d_{N}\end{array}\right]^{T}$ based on some criterion. In a conventional VP, such as the ZF VP [11] or the MMSE VP [12], the effective symbol vector $\mathbf{d}$ is expressed as

$$
\mathbf{d}=\mathbf{P}(\mathbf{x}+\boldsymbol{\omega}),
$$

where $\mathbf{P}$ is the $(N \times K)$-element precoding matrix and $\boldsymbol{\omega}$ is the $K$-element discrete-valued perturbation vector, our design objective is to determine $\mathbf{P}$ and $\boldsymbol{\omega}$ separately based on $\mathbf{H}$ and $\mathbf{x}$. Our proposed scheme, however, determines $\mathbf{d}$ directly and therefore it is referred to as the generalised VP.

The DL channel's white noise vector is defined by $\mathbf{n}=$ $\left[\begin{array}{ll}n_{1} & n_{2} \cdots n_{K}\end{array}\right]^{T}$, where $n_{k}, 1 \leq k \leq K$, is complex-valued Gaussian distributed with a zero mean and $E\left[\left|n_{k}\right|^{2}\right]=2 \sigma_{n}^{2}=$ $\mathrm{N}_{\mathrm{O}}$. Given a fixed total transmit power $\mathrm{E}_{\mathrm{T}}$ at the $\mathrm{BS}$, an appropriate scaling factor is defined by $\alpha=\sqrt{\mathrm{E}_{\mathrm{T}} /\|\mathbf{d}\|^{2}}$, which is used to fullfill this power constraint. At the receiver, the reciprocal of the scaling factor, namely $\alpha^{-1}$, is used to scale the received signal in order to maintain unity-gain transmission. The energy per bit per antenna is defined by $\mathrm{E}_{\mathrm{b}}=\mathrm{E}_{\mathrm{T}} / N \log _{2} M$ for $M$-ary modulation. The received signal vector $\hat{\mathbf{y}}=\left[\begin{array}{ll}\hat{y}_{1} & \hat{y}_{2} \cdots \hat{y}_{K}\end{array}\right]^{T}$ recorded at the input of the modulo operation of Fig. 1 is given by

$$
\hat{\mathbf{y}}=\mathbf{H}^{T} \mathbf{d}+\alpha^{-1} \mathbf{n} .
$$

The modulo operation invoked for $\hat{y}_{k}$ is described by

$$
\bmod _{\tau}\left(\hat{y}_{k}\right)=\hat{y}_{k}-\left\lfloor\frac{\Re\left[\hat{y}_{k}\right]+\tau / 2}{\tau}\right\rfloor \tau-j\left\lfloor\frac{\Im\left[\hat{y}_{k}\right]+\tau / 2}{\tau}\right\rfloor \tau \text {. }
$$

where $1 \leq k \leq K, \Re[\bullet]$ and $\Im[\bullet]$ denote the real and imaginary parts, respectively, $j^{2}=-1,\lfloor\bullet\rfloor$ denotes the integer floor operator, and $\tau$ is a positive number determined by the modulation constellation employed. The role of the modulo operation was graphically portrayed in [11] and it is detailed further below. The received signal vector $\mathbf{y}=\left[\begin{array}{ll}y_{1} & y_{2} \cdots y_{K}\end{array}\right]^{T}$ at the output of the modulo operation of Fig. 1 is given by

$$
\mathbf{y}=\bmod _{\tau}(\hat{\mathbf{y}}),
$$

and $y_{k}, 1 \leq k \leq K$, constitutes sufficient statistics for the $k$ th MS to detect the transmitted information data symbol $x_{k}$. The authors of [11] suggested to choose $\tau$ according to

$$
\tau=2\left(|c|_{\max }+\Delta / 2\right),
$$

where $|c|_{\max }$ is the largest distance of the modulated symbols to the real or imaginary axis, and $\Delta$ is the spacing between the constellation points. Specifically, we consider 4 level quadrature amplitude modulation (4-QAM), where all the four symbols have the amplitude of $\sqrt{2} / 2$. The values of $|c|_{\max }$ and $\Delta$ are given by $|c|_{\max }=\frac{1}{2}$ and $\Delta=1$, respectively. Thus we have $\tau=2$ according to (6). The modulo operator (4) maps the received signal, $\Re\left[\hat{y}_{k}\right]$ and $\Im\left[\hat{y}_{k}\right]$, into the interval $[-\tau / 2, \tau / 2)$. For example, if we have $\Re\left[\hat{y}_{k}\right]=1.75 \tau$, it is then mapped to $\Re\left[y_{k}\right]=-0.25 \tau$ by the modulo operation.

\section{Generalised MBER Vector Precoder Design}

For notational simplicity, we restrict our treatment to the 4QAM case of $M=4$, noting that its extension to a high-order QAM scheme can be achieved by considering the minimum symbol error rate (MSER) criterion, which was employed for a MUD in [20]. The BER encountered at the receiver's output after the modulo operation for the in-phase component of user $k$ is

$$
P_{e_{I}, k}(\mathbf{d})=\operatorname{Prob}\left\{\operatorname{sgn}\left(\Re\left[x_{k}\right]\right) \Re\left[y_{k}\right]<0\right\} .
$$

Let us now define the signed decision variable $s_{k}=\operatorname{sgn}\left(\Re\left[x_{k}\right]\right) \Re\left[\hat{y}_{k}\right]$, which has a mean of $c_{R}^{(k)}=\operatorname{sgn}\left(\Re\left[x_{k}\right]\right) \Re\left[\mathbf{h}_{k}^{T} \mathbf{d}\right]$ and the probability density function (PDF) given by

$$
p\left(s_{k}\right)=\frac{1}{\sqrt{2 \pi} \alpha^{-1} \sigma_{n}} e^{-\frac{\left(s_{k}-c_{R}^{(k)}\right)^{2}}{2 \sigma_{n}^{2} \alpha^{-2}}} .
$$

The decision areas are periodically extended in the $s_{k}$-axis, as depicted in Fig. 2, where the intervals marked by - are the error areas of $\operatorname{sgn}\left(\Re\left[x_{k}\right]\right) \Re\left[y_{k}\right]<0$, while the intervals marked by + are the error-free areas of $\operatorname{sgn}\left(\Re\left[x_{k}\right]\right) \Re\left[y_{k}\right]>0$. An error occurs, when $s_{k}$ falls into the intervals $\left[\frac{2 m+1}{2} \tau,(m+1) \tau\right)$, $-\infty<m<\infty$. Therefore, $P_{e_{I}, k}(\mathbf{d})$ is given by

$$
\begin{gathered}
P_{e_{I}, k}(\mathbf{d})=\sum_{m=-\infty}^{\infty} \int_{\frac{2 m+1}{2} \tau}^{(m+1) \tau} p\left(s_{k}\right) d s_{k} \approx \int_{-\infty}^{-3 \tau} p\left(s_{k}\right) d s_{k} \\
+\int_{-\frac{5 \tau}{2}}^{-2 \tau} p\left(s_{k}\right) d s_{k}+\int_{-\frac{3 \tau}{2}}^{-\tau} p\left(s_{k}\right) d s_{k}+\int_{-\frac{\tau}{2}}^{0} p\left(s_{k}\right) d s_{k} \\
+\int_{\frac{\tau}{2}}^{\tau} p\left(s_{k}\right) d s_{k}+\int_{\frac{3 \tau}{2}}^{2 \tau} p\left(s_{k}\right) d s_{k}+\int_{\frac{5 \tau}{2}}^{3 \tau} p\left(s_{k}\right) d s_{k},
\end{gathered}
$$

where the approximation occurs, when we lump all the integrations over all the error intervals in $(-\infty,-3 \tau)$ and $(3 \tau, \infty)$ together into a single integration over the interval of $(-\infty,-3 \tau)$. This approximation is fairly accurate owing to the near-symmetry of the PDF (8) over the two regions of $(3 \tau, \infty)$ and $(-\infty,-3 \tau)$. Furthermore the last six integrates at the righthand side of the approximation are generally higher than the first term. Thus $P_{e_{I}, k}(\mathbf{d})$ can be approximated as

$$
\begin{aligned}
P_{e_{I}, k}(\mathbf{d}) & \approx Q\left(\frac{c_{R}^{(k)}+3 \tau}{\alpha^{-1} \sigma_{n}}\right)+Q\left(\frac{-\frac{5 \tau}{2}-c_{R}^{(k)}}{\alpha^{-1} \sigma_{n}}\right) \\
- & Q\left(\frac{-2 \tau-c_{R}^{(k)}}{\alpha^{-1} \sigma_{n}}\right)+Q\left(\frac{-\frac{3 \tau}{2}-c_{R}^{(k)}}{\alpha^{-1} \sigma_{n}}\right) \\
- & Q\left(\frac{-\tau-c_{R}^{(k)}}{\alpha^{-1} \sigma_{n}}\right)+Q\left(\frac{-\frac{\tau}{2}-c_{R}^{(k)}}{\alpha^{-1} \sigma_{n}}\right)
\end{aligned}
$$




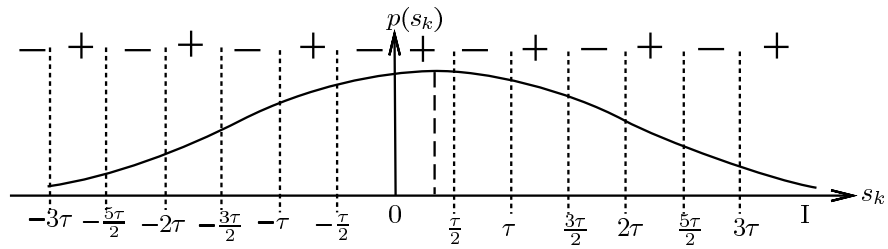

Fig. 2. Probability density function of the decision variable $s_{k}$.

$$
\begin{aligned}
& -Q\left(\frac{-c_{R}^{(k)}}{\alpha^{-1} \sigma_{n}}\right)+Q\left(\frac{\frac{\tau}{2}-c_{R}^{(k)}}{\alpha^{-1} \sigma_{n}}\right)-Q\left(\frac{\tau-c_{R}^{(k)}}{\alpha^{-1} \sigma_{n}}\right) \\
& +Q\left(\frac{\frac{3 \tau}{2}-c_{R}^{(k)}}{\alpha^{-1} \sigma_{n}}\right)-Q\left(\frac{2 \tau-c_{R}^{(k)}}{\alpha^{-1} \sigma_{n}}\right) \\
& +Q\left(\frac{\frac{5 \tau}{2}-c_{R}^{(k)}}{\alpha^{-1} \sigma_{n}}\right)-Q\left(\frac{3 \tau-c_{R}^{(k)}}{\alpha^{-1} \sigma_{n}}\right) .
\end{aligned}
$$

Hence, the average BER of the in-phase component of $\mathbf{y}$ is

$$
P_{e_{I}, \mathbf{x}}(\mathbf{d})=\frac{1}{K} \sum_{k=1}^{K}\left(P_{e_{I}, k}(\mathbf{d})\right) .
$$

Similarly, let $c_{I}^{(k)}=\operatorname{sgn}\left(\Im\left[x_{k}\right]\right) \Im\left[\mathbf{h}_{k}^{T} \mathbf{d}\right]$. Then the BER of the quadrature-phase component for the $k$ th user is given by

$$
\begin{aligned}
P_{e_{Q}, k}(\mathbf{d}) & \approx Q\left(\frac{c_{I}^{(k)}+3 \tau}{\alpha^{-1} \sigma_{n}}\right)+Q\left(\frac{-\frac{5 \tau}{2}-c_{I}^{(k)}}{\alpha^{-1} \sigma_{n}}\right) \\
- & \left(\frac{-2 \tau-c_{I}^{(k)}}{\alpha^{-1} \sigma_{n}}\right)+Q\left(\frac{-\frac{3 \tau}{2}-c_{I}^{(k)}}{\alpha^{-1} \sigma_{n}}\right) \\
- & \left(\frac{-\tau-c_{I}^{(k)}}{\alpha^{-1} \sigma_{n}}\right)+Q\left(\frac{-\frac{\tau}{2}-c_{I}^{(k)}}{\alpha^{-1} \sigma_{n}}\right) \\
- & \left(\frac{-c_{I}^{(k)}}{\alpha^{-1} \sigma_{n}}\right)+Q\left(\frac{\frac{\tau}{2}-c_{I}^{(k)}}{\alpha^{-1} \sigma_{n}}\right)-Q\left(\frac{\tau-c_{I}^{(k)}}{\alpha^{-1} \sigma_{n}}\right) \\
+ & \left(\frac{\frac{3 \tau}{2}-c_{I}^{(k)}}{\alpha^{-1} \sigma_{n}}\right)-Q\left(\frac{2 \tau-c_{I}^{(k)}}{\alpha^{-1} \sigma_{n}}\right) \\
+ & \left(\frac{\frac{5 \tau}{2}-c_{I}^{(k)}}{\alpha^{-1} \sigma_{n}}\right)-Q\left(\frac{3 \tau-c_{I}^{(k)}}{\alpha^{-1} \sigma_{n}}\right) .
\end{aligned}
$$

Then the average BER for the quadrature-phase component of $\mathbf{y}$ at the receivers of the $K \mathrm{MSs}$ is given by

$$
P_{e_{Q}, \mathbf{x}}(\mathbf{d})=\frac{1}{K} \sum_{k=1}^{K}\left[P_{e_{Q}, k}(\mathbf{d})\right] .
$$

Thus, the average BER for 4-QAM signalling becomes

$$
P_{e, \mathbf{x}}(\mathbf{d})=\left[P_{e_{I}, \mathbf{x}}(\mathbf{d})+P_{e_{Q}, \mathbf{x}}(\mathbf{d})\right] / 2 .
$$

Hence, the optimal continuous-valued effective symbol vector $\mathbf{d}_{\text {opt }}$ is found by solving the following optimisation problem

$$
\mathbf{d}_{\mathrm{opt}}=\arg \min _{\mathbf{d}} P_{e, \mathbf{x}}(\mathbf{d}) \text {. }
$$

Note that (15) constitutes a non-convex continuous-valued optimisation problem. Hence we invoke the powerful PSO algorithm [17], [18] to solve this generalised MBER VP design problem. The detailed algorithm is now described.
A swarm of particles, $\left\{\mathbf{d}_{i}^{(l)}\right\}_{i=1}^{S}$, that represent potential solutions are evolved, a set of initial solutions to the find solution in the search space $\mathrm{D}^{K}$, where $S$ is the swarm size, the index $l$ denotes the iteration step-size and

$$
\mathrm{D}=\left[-P_{\max }, P_{\max }\right]+j\left[-P_{\max }, P_{\max }\right]
$$

specifies the search range for each element of $\mathbf{d}$.

a) Initialisation. For $l=0$, we choose $\mathbf{d}_{1}^{(l)}$ to be the solution of the improved MMSE VP scheme [16], and generate the rest of the particles, $\left\{\mathbf{d}_{i}^{(l)}\right\}_{i=2}^{S}$, randomly in the search space $\mathrm{D}^{K}$. b) Evaluation. Each particle $\mathbf{d}_{i}^{(l)}$ has an associated cost $P_{e, \mathbf{x}}\left(\mathbf{d}_{i}^{(l)}\right)$. Each particle $\mathbf{d}_{i}^{(l)}$ remembers its best visited position, denoted as $\mathbf{p b}_{i}^{(l)}$, which provides the cognitive information. Every particle also knows the best position visited by any particle throughout the entire swarm, denoted as $\mathbf{g b}^{(l)}$, which provides the social information. The cognitive information $\left\{\mathbf{p b}_{i}^{(l)}\right\}_{i=1}^{S}$ and the social information $\mathbf{g b}^{(l)}$ are updated at each iteration, given the new cost information $\left\{P_{e, \mathbf{x}}\left(\mathbf{d}_{i}^{(l)}\right)\right\}_{i=1}^{S}$.

c) Update. Each particle $\mathbf{d}_{i}^{(l)}$ has a velocity $\mathbf{v}_{i}^{(l)}$ used for directing its "flight" or search. both the velocity and position of the $i$ th particle are updated in each iteration according to:

$$
\begin{aligned}
\mathbf{v}_{i}^{(l+1)}= & w_{\mathrm{I}} * \mathbf{v}_{i}^{(l)}+c_{1} * \varphi_{1} *\left(\mathbf{p} \mathbf{b}_{i}^{(l)}-\mathbf{d}_{i}^{(l)}\right) \\
& +c_{2} * \varphi_{2} *\left(\mathbf{g} \mathbf{b}^{(l)}-\mathbf{d}_{i}^{(l)}\right), \\
\mathbf{d}_{i}^{(l+1)}= & \mathbf{d}_{i}^{(l)}+\mathbf{v}_{i}^{(l+1)},
\end{aligned}
$$

where $w_{\mathrm{I}}$ is the inertia weight, $c_{1}$ and $c_{2}$ are the two acceleration coefficients, while $\varphi_{1}=\operatorname{rand}()$ and $\varphi_{2}=\operatorname{rand}()$ denotes the two random variables uniformly distributed in $(0,1)$.

In order to avoid excessive by diverse roaming of particles beyond the search space, a velocity space $\mathrm{V}^{K}$ obeying

$$
\mathrm{V}=\left[-V_{\max }, V_{\max }\right]+j\left[-V_{\max }, V_{\max }\right]
$$

is imposed, so that each element of $\mathbf{v}_{i}^{(l+1)}$ is forced to remain within the velocity range $V$ defined in (19). Similarly, if a particle $\mathbf{d}_{i}^{(l+1)}$ moves outside the search space, it is moved back into the search space to a random position.

d) Termination. If the maximum number of iterations, $I_{\mathrm{m}}$, is reached, we terminate the algorithm at the final solution of $\mathbf{d}_{\mathrm{opt}}=\mathbf{g b}^{\left(I_{\mathrm{m}}\right)}$; otherwise, we set $l=l+1$ and go to Step $b$ ).

The complexity of this PSO aided generalised MBER-VP design is characterised in Table I, in comparison to the complexity of the MMSE-VP design [12]. In Step a), one of the particles is initialised to the solution of the improved MMSEVP [16]. Then it may be readily shown that the complexity of this initialisation is proportioned to $(73 K+18 K N+6 N+4) D$, where $D$ is the number of extended constellation points visited [12], [16].

TABLE I

COMPUTATIONAL COMPLEXITY OF THE MMSE-VP [12] AND THE PROPOSED MBER-VP DESIGNS FOR 4-QAM SIGNALLING.

\begin{tabular}{|c|l|}
\hline Algorithm & Flops \\
\hline MMSE-VP & $\left(\frac{7}{3} K^{3}+13 K^{2}+13 K-1\right) D+\mathcal{O}\left(9 K^{2} N^{2}-2 K^{2}\right)$ \\
\hline MBER-VP & $\begin{array}{l}\left(7 N+(73+7 N) K+(23 N+(73+7 N) K+6) I_{\mathrm{m}}\right) S \\
+(73 K+18 K N+6 N+4) D+2 K+7 K N+5 S\end{array}$ \\
\hline
\end{tabular}




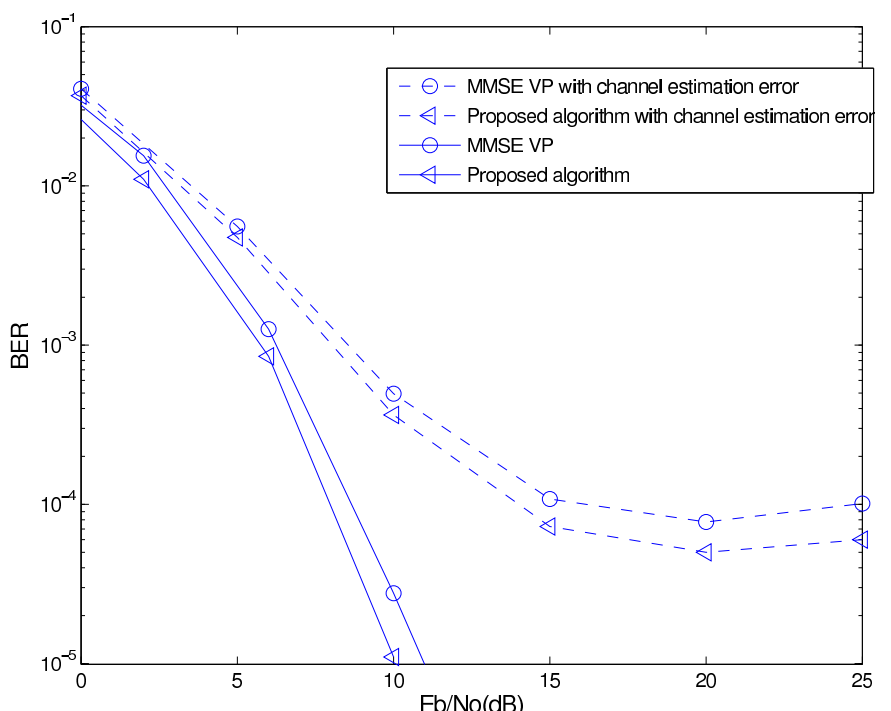

Fig. 3. BER versus SNR performance of the MMSE VP [12] and our proposed MBER generalised VP for communicating over flat Rayleigh fading channels using $N=4$ transmit antennas to support $K=44$-QAM users.

The size of the search space (16) is specified by the specific optimisation problem considered. For 4-QAM signalling, our extensive empirical results suggest that the magnitudes of $\Re\left[d_{k}\right]$ or $\Im\left[d_{k}\right]$ obtained by the MMSE VP solution are typically lower than 1.0, and they may only occassionally be slightly larger than 1.0. This observation is also true for the proposed generalised MBER VP design. Therefore, we set $P_{\max }=1.2$. The velocity limit $V_{\max }$ is typically related to $P_{\max }$, and we empirically set $V_{\max }=0.2$. The inertia weight was chosen as $w_{\mathrm{I}}=\operatorname{rand}()$, which was found to perform better in our investigations not included here owing to lack of space than the alternative choice of setting $w_{\text {I }}$ to a small positive constant or to zero. The time-varying acceleration coefficients were defined in [19], and $c_{1}$ was varied from 2.5 to 0.5 , while $c_{2}$ varied from 0.5 to 2.5 during the iterative procedure. Alternatively, setting them to

$$
\begin{aligned}
& c_{1}=(0.5-2.5) \cdot l / I_{\mathrm{m}}+2.5, \\
& c_{2}=(2.5-0.5) \cdot l / I_{\mathrm{m}}+0.5,
\end{aligned}
$$

also works well in our application. The appropriate values of $S$ and $I_{\mathrm{m}}$ have to be carefully chosen to ensure that the algorithm converges to the optimal solution at a modest computational complexity.

\section{Simulation Results}

The proposed PSO aided generalised MBER VP design was investigated using the MMSE VP design [12] as the benchmark. We considered the DL of a multiuser system employing $N$ transmit antennas at the BS to support $K 4$ QAM MSs. The received signals after the modulo operation of Fig. 1 were directly used for making decisions. The signalto-noise ratio (SNR) was defined as $\mathrm{SNR}=\mathrm{E}_{\mathrm{b}} / \mathrm{N}_{\mathrm{o}}$. All the simulation results were averaged over 100 channel realisations. The appropriate swarm size was found empirically to be $S=20$, and the maximum number of iterations ranged from $I_{\mathrm{m}}=20$ to 45 , depending on both the specific system and on the SNR value.
We considered the case of $N=4$ and $K=4$. First, the perfect knowledge of the DL CIR matrix was assumed to be available at the BS. Fig. 3 shows that the proposed generalised MBER VP scheme achieved $1 \mathrm{~dB}$ SNR gain at the target BER of $10^{-5}$ over the MMSE VP. Fig. 4 indicates that the PSO algorithm converged after $I_{\mathrm{m}}=20$ and 40 for the SNR values of $6 \mathrm{~dB}$ and $10 \mathrm{~dB}$, respectively. The robustness of the two VP algorithms against the channel estimation error was investigated next. Complex-valued Gaussian white noise having a variance of 0.01 was added to each channel tap $h_{i, k}$ in order to represent the channel estimation error, and the BERs of these two nonlinear MUT designs contaminated by this channel estimation error were also depicted in Fig. 3, where it can be seen that the generalised MBER VP had a similar sensitivity to channel estimation errors to that of the MMSE VP design.

The system was then configured to use $N=2$ transmit antennas to support $K=4$ 4-QAM users, which was a strongly rank-deficient scenario. The BERs of the two algorithms are shown in Fig. 5 assuming the perfect knowledge of the channel matrix as well as assuming the same channel estimation error as specified in the previous example. Even the powerful nonlinear MMSE VP suffered from an error floor in this demanding scenario. By contrast, the generalised MBER VP did not exhibit an error floor, which demonstrated its ability to operate successfully in the challenging rank-deficient scenario considered. Fig. 4 shows that the PSO algorithm converged after $I_{\mathrm{m}}=40$ and 45 , respectively, for the SNRs of $25 \mathrm{~dB}$ and $30 \mathrm{~dB}$, respectively. The computational complexity imposed and the run times recorded by the two VP designs are compared in Table II, where it can be clearly seen that the total complexity of the PSO aided generalised MBER VP design is less than twice of the conventional MMSE-VP design. Fig. 6 shows that $S=10$ was insufficient for the PSO algorithm, while the PSO algorithms having $S=20,30$ and 40 all converged to the optimal solution. However, the choice

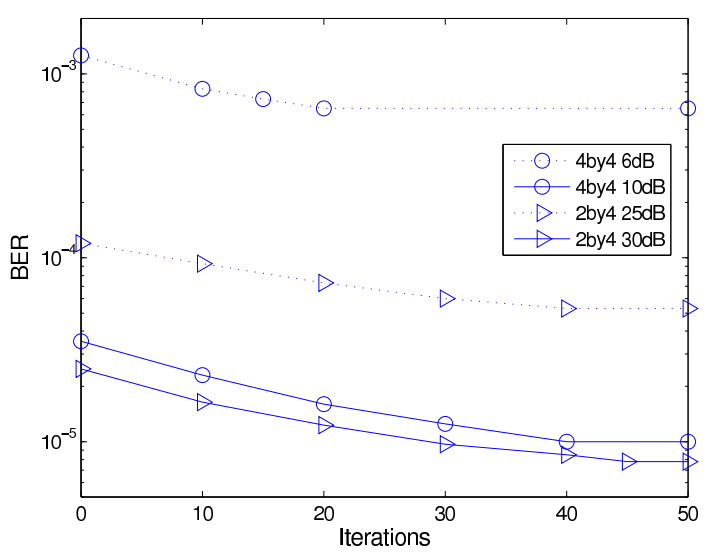

Fig. 4. Convergence of the PSO aided MBER generalised VP algorithm given different SNR values for communicating over flat Rayleigh fading $4 \times 4$ and $2 \times 4$ systems, respectively, where the swarm size is $S=20$.

FLOPS AND RUN TIMES REQUIRED BY THE MMSE-VP AND MBER-VP DESIGNS FOR THE RANK-DEFICIENT SYSTEM, GIVEN TWO SNR VALUES.

\begin{tabular}{|c|c|l|}
\hline Algorithm & Flops/Time $(\mathrm{SNR}=25 \mathrm{~dB})$ & Flops/Time $(\mathrm{SNR}=30 \mathrm{~dB})$ \\
\hline MMSE-VP & $2,508,638 / 4787.3(\mathrm{~s})$ & $2,609,600 / 4981.9(\mathrm{~s})$ \\
\hline MBER-VP & $4,064,937 / 8878.9(\mathrm{~s})$ & $4,471,060 / 9565.8(\mathrm{~s})$ \\
\hline
\end{tabular}




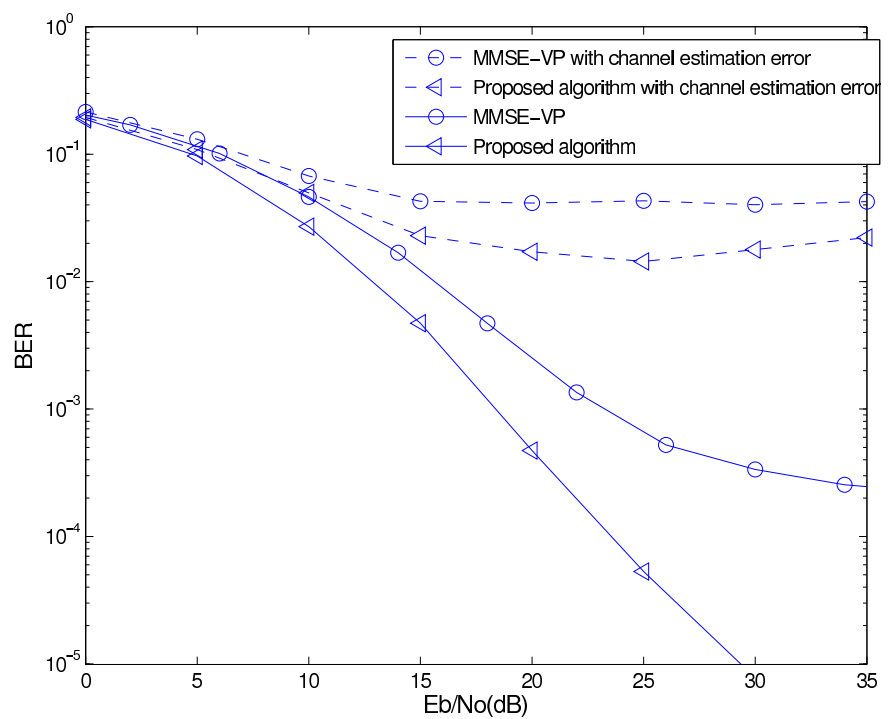

Fig. 5. BER versus SNR performance of the MMSE VP [12] and our proposed MBER generalised VP for communicating over flat Rayleigh fading channels using $N=2$ transmit antennas to support $K=44$-QAM users.

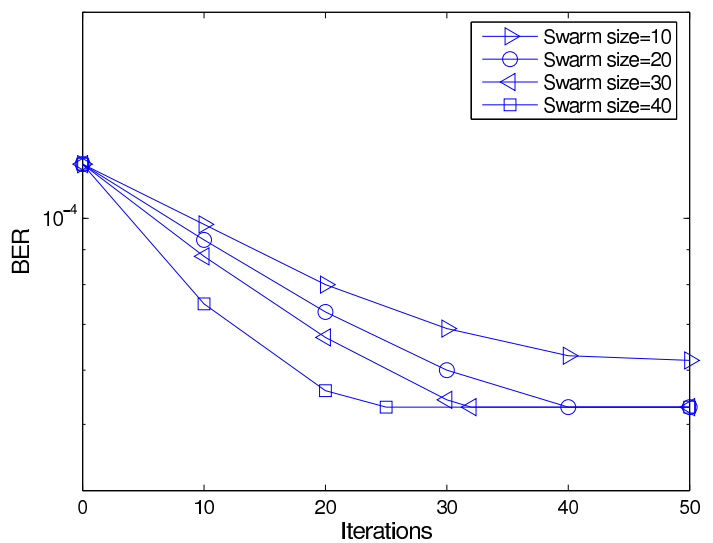

Fig. 6. Convergence of the PSO aided MBER generalised VP algorithm with different swarm sizes $S$ for for the rank-deficient system given the SNR value of $25 \mathrm{~dB}$.

of $S=20$ was attractive owing to its low complexity, as seen from Table III.

\section{CONCLUSiONS}

We have proposed a generalised MBER VP scheme for the DL of a multiuser system, where the BS equipped with multiple transmit antennas communicates with single-antenna aided non-cooperative MS receivers each equipped with a modulo device. Our PSO aided nonlinear transmit preprocessing scheme generates the effective symbol vector based on the MBER criterion with the knowledge of both the downlink CIR matrix and the current information symbol vector to be transmitted. Our PSO aided generalised MBER VP scheme outperforms even the powerful nonlinear MMSE VP scheme,

TABLE III

COMPLEXITY OF THE PSO AIDED MBER-VP DESIGN FOR THE RANK-DEFICIENT SYSTEM, GIVEN SNR $=25 \mathrm{DB}$.

\begin{tabular}{|c|c|c|}
\hline Swarm size $S$ & number of iterations $I_{\mathrm{m}}$ & complexity (Flops) \\
\hline 20 & 40 & $4,064,937$ \\
30 & 32 & $4,149,627$ \\
40 & 25 & $4,174,077$ \\
\hline
\end{tabular}

especially in the challenging rank-deficient scenario, while imposing less than twice higher computational complexity.

\section{REFERENCES}

[1] L.-L. Yang, "Design of linear multiuser transmitters from linear multiuser receivers," in Proc. ICC 2007, (Glasgow, UK), pp. 5258-5263, June 24-28, 2007.

[2] B. R. Vojčić and W. M. Jang, "Transmitter precoding in synchronous multiuser communications," IEEE Trans. Communications, vol. 46, pp. 1346-1355, October 1998.

[3] D. Yang, L.-L. Yang and L. Hanzo, "Performance of SDMA systems using transmitter preprocessing based on noisy feedback of vector-quantized channel impulse responses," in Proc. VTC2007-Spring, (Dublin, Ireland), pp. 2119-2123, April 22-25, 2007.

[4] R. Irmer, R. Habendorf, W. Rave and G. Fettweis, "Nonlinear multiuser transmission using multiple antennas for TDD-CDMA," in Proc. 6th Int. Symp. Wireless Personal Multimedia Communications, (Yokosuka, Japan), pp. 251-255, October 19-22, 2003.

[5] R. Habendorf and G. Fettweis, "Nonlinear optimization for the multiuser downlink," in Proc. 13th European Wirelss Conf., (Paris, France), April 1-4, 2007.

[6] F. Richter, A. Fischer, R. Habendorf and G. Fettweis, "Transmitter-based minimization of error rates in the downlink of wireless systems," in Proc. GLOBECOM 2008, (New Orleans, Louisiana), Nov.30-Dec.04, 2008.

[7] A. Hjørungnes and P. S. R. Diniz, "Minimum BER prefilter transform for communications systems with binary signaling and known FIR MIMO channel," IEEE Signal Processing Letters, vol. 12, pp. 234-237, March 2005.

[8] S. Tan, Minimum Error Rate Beamforming Transceivers. PhD thesis, School of Electronics and Computer Science, University of Southampton, UK, April 2008.

[9] W. Yao, S. Chen, S. Tan and L. Hanzo, "Particle swarm optimisation aided minimum bit error rate multiuser transmission," in Proc. ICC 2009, (Dresden, Germany), p. 5 pages, June 14-18, 2009.

[10] M. Costa, "Writing on dirty paper," IEEE Trans. Information Theory, vol. 29, pp. 439-441, May 1983.

[11] B. M. Hochwald, C. B. Peel and A. L. Swindlehurst, "A vectorperturbation technique for near-capacity multiantenna multiuser communication - part II: perturbation," IEEE Trans. Communications, vol. 53, pp. 537-544, March 2005.

[12] D. A. Schmidt, M. Joham and W. Utschick, "Minimum mean square error vector precoding," in Proc. PIMRC 2005, vol. 1, (Berlin, Germany), pp. 107-111, Sept.11-14 2005.

[13] E. Y. Kim and J. Chun, "Optimum vector perturbation minimizing total MSE in multiuser MIMO downlink," in Pro. ICC 2006, vol. 9, (Istanbul, Turkey), pp. 4242-4247, June 11-15, 2006.

[14] W. S. Chua, C. Yuen and F. Chin, "A continuous vector-perturbation for multi-antenna multi-user communication," in Proc. VTC2007-Spring, (Dublin, Ireland), pp. 1806-1810, April 22-25, 2007.

[15] C. Yuen and B. M. Hochwald, "How to gain $1.5 \mathrm{~dB}$ in vector precoding," in Proc. Globecom 2006, (San Francisco, USA), p. 5 pages, Nov.27Dec.1, 2006.

[16] W. Yao, S. Chen and L. Hanzo, "Improved MMSE vector precoding based on the MBER criterion," in Proc. VTC2009-Spring, (Barcelona, Spain), p. 5 pages, April 26-29, 2009.

[17] J. Kennedy and R. Eberhart, "Particle swarm optimization," in Proc. of 1995 IEEE Int. Conf. Neural Networks, vol. 4, (Perth, Australia), pp. 1942-1948, Nov. 27 - Dec. 1, 1995.

[18] J. Kennedy and R. C. Eberhart, Swarm Intelligence. Morgan Kaufmann, 2001.

[19] A. Ratnaweera, S. K. Halgamuge and H. C. Watson, "Self-organizing hierarchical particle swarm optimizer with time-varying acceleration coefficients," IEEE Trans. Evolutionary Computation, vol. 8, pp. 240255, June 2004.

[20] S. Chen, A. Livingstone, H.-Q. Du and L. Hanzo, "Adaptive minimum symbol error rate beamforming assisted detection for quadrature amplitude modulation," IEEE Trans. Wireless Communications, vol. 7, pp. 1140-1145, April 2008. 